\title{
铜基催化剂表面电催化碳-碳耦合的机理研究
}

陆奇

清华大学化学工程系, 北京 100084

\section{Mechanistic Insights into Electroreductive C-C Coupling on $\mathrm{Cu}$}

Qi Lu

Department of Chemical Engineering, Tsinghua University, Beijing 100084, China.

Email: luqicheme@mail.tsinghua.edu.cn

Published online: January 4, 2021.

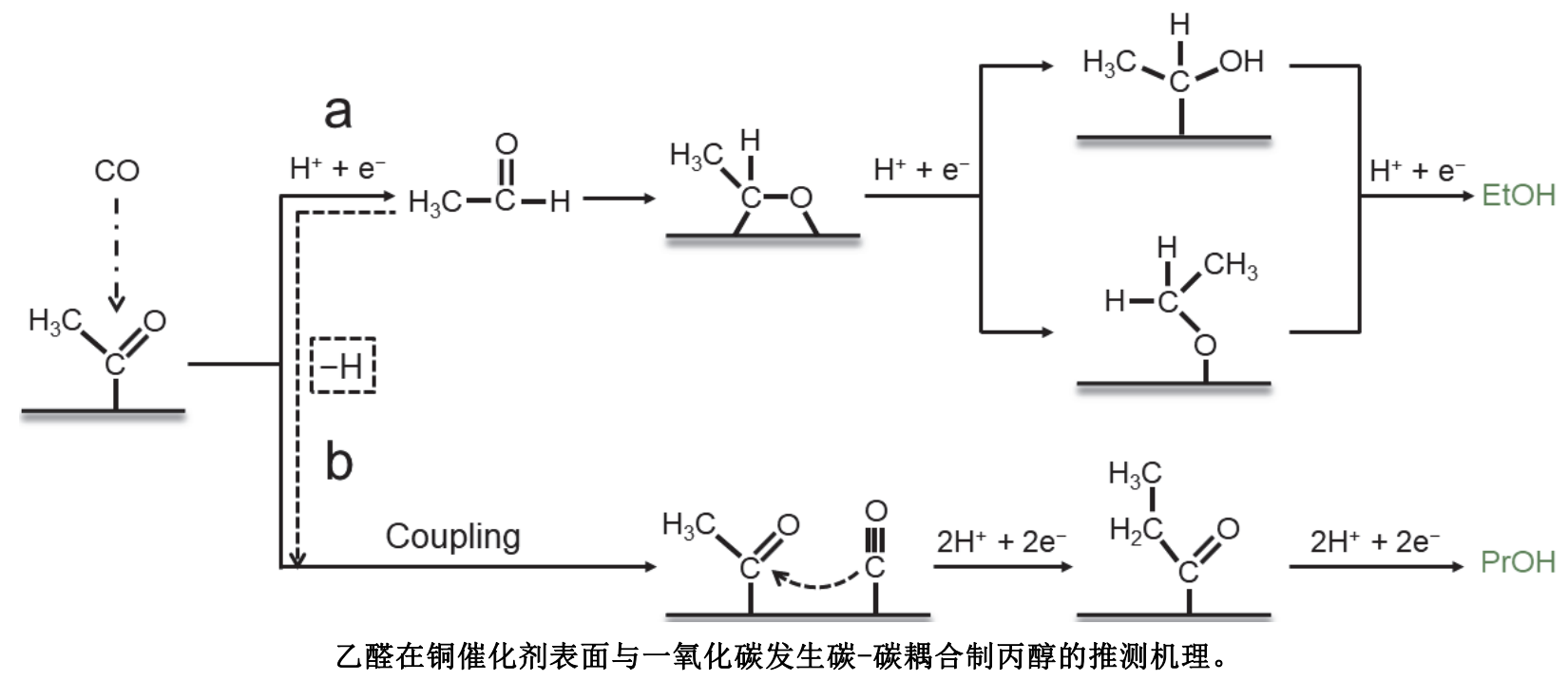

电催化 $\mathrm{CO}_{2}$ 还原, 及其关键中间体 $\mathrm{CO}$ 的还原, 以制备高价值的含碳产物, 有助于人工碳循环的 构建以及人类的可持续发展 1,2 。其中, 碳-碳耦合 过程的促进对高价值多碳产物的选择性制备具有 十分重要的意义 3,4 。铜基催化剂具有将 $\mathrm{CO}_{2}$ 或 $\mathrm{CO}$ 选择性转化为多碳产物的独特性能 ${ }^{2}$, 因此对铜表 面电催化碳-碳耦合机理的研究至关重要。

近些年电催化 $\mathrm{CO}_{2} / \mathrm{CO}$ 还原领域的研究主要 集中在催化剂结构及反应体系的设计和优化, 以

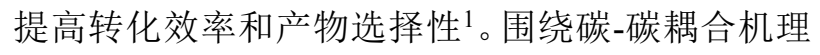
的研究则集中于铜表面两个吸附态 CO之间的耦 合过程, 以研究 $\mathrm{C}_{2}$ 产物的生成机理 6,7 。例如, 研究 发现乙醛是 $\mathrm{CO}_{2} / \mathrm{CO}$ 还原反应过程中的重要反应 中间体, 并为最终产物乙醇的前驱体。而对于 $\mathrm{C}_{3+}$
产物(例如正丙醇)生成过程中的碳一碳耦合机理的 研究还较为缺乏, 但其对催化剂、反应体系的理性 设计和优化, 以高效制备更富价值的 $\mathrm{C}_{3+}$ 产物具有 十分重要的意义 8

为探究 $\mathrm{C}_{3}$ 产物生成过程中的碳一碳耦合机 理, 美国特拉华大学徐冰君研究组利用同位素示 踪结合原位红外光谱的分析, 考察了 $\mathrm{CO}$ 与人为加 入的乙醛在铜表面耦合制备正丙醇的反应过程, 并系统研究了 $\mathrm{CO}$ 与不同浓度乙醛之间的耦合程 度, 据此推测了该过程中 $\mathrm{C}_{1}$ 与 $\mathrm{C}_{2}$ 间的耦合机理。

他们的实验结果表明, 在 $\mathrm{CO}$ 还原反应中加入 乙醛分子, 将会抑制正丙醇的生成; 然而如果在 ${ }^{13} \mathrm{CO}$ 还原反应中加入乙醛 $\left(\mathrm{CH}_{3} \mathrm{CHO}\right),{ }^{13} \mathrm{CO}$ 将与乙 醛中的羰基碳 $(-\mathrm{CHO})$ 耦合并生成产物正丙醇中 
的羟甲基(即生成 $\mathrm{CH}_{3} \mathrm{CH}_{2}{ }^{13} \mathrm{CH}_{2} \mathrm{OH}$ ), 证明了 $\mathrm{CO}$ 与 乙醛间存在的耦合反应。基于此, 他们进一步考察 了 $\mathrm{CO}$ 与氛代乙醛 $\left(\mathrm{CD}_{3} \mathrm{CDO}\right)$ 之间的耦合反应, 通 过不同浓度 $\mathrm{CD}_{3} \mathrm{CDO}$ 的加入, 证明了即使在高浓度 乙醛(高达 $40 \mathrm{mmol} \cdot \mathrm{L}^{-1}$ ) 存在的情况下, 仅有 $36 \%$ 的 正丙醇产自 $\mathrm{CO}$ 与乙醛的耦合反应, 其余大部分的 正丙醇仍然产自 $\mathrm{CO}$ 分子之间的自耦合反应, 证明 了乙醛分子并不是 $\mathrm{CO}_{2} / \mathrm{CO}$ 还原反应中正丙醇生 成的前驱体。同时, 在加入乙酫后有大量乙醇生成 的事实基础上, 他们进一步结合原位表面增强红 外光谱中, 加入乙醛分子后表面吸附 $\mathrm{C}-\mathrm{O}$ 键的发 现, 推测了乙醛还原生成乙醇, 以及 $\mathrm{CO}$ 与乙醛耦 合生成正丙醇的反应机理。在该机理中, 乙醛脱氢 吸附的产物 $\mathrm{CH}_{3} \mathrm{CO}$-被推测为可与 $\mathrm{CO}$ 耦合生成 正丙醇的反应中间体。

上述研究工作发表在Journal of the American Chemical Society 上 ${ }^{9}$ 。此项工作揭示了 $\mathrm{CO}_{2} / \mathrm{CO}$ 还原 反应中 $\mathrm{C}_{3}$ 产物生成过程中的碳-碳耦合机理, 为设 计高效制备 $\mathrm{C}_{3}$ 产物的催化剂提供了参考思路。

\section{References}

(1) Nitopi, S.; Bertheussen, E.; Scott, S. B.; Liu, X.; Engstfeld, A. K.;
Horch, S.; Seger, B.; Stephens, I. E. L.; Chan, K.; Hahn, C.; et al. Chem. Rev. 2019, 119, 7610. doi: 10.1021/acs.chemrev.8b00705

(2) Chang, X.; Wang, T.; Gong, J. Energy Environ. Sci. 2016, 9, 2177. doi: $10.1039 / \mathrm{c} 6 \mathrm{ee} 00383 \mathrm{~d}$

(3) Hori, Y.; Takahashi R.; Yoshinami Y.; Murata A. J. Phys. Chem. B 1997, 101, 7075. doi: 10.1021/jp970284i

(4) Wuttig, A.; Liu, C.; Peng, Q.; Yaguchi, M.; Hendon, C. H.; Motobayashi, K.; Ye, S.; Osawa, M.; Surendranath, Y. ACS Cent. Sci. 2016, 2, 522. doi: 10.1021/acscentsci.6b00155

(5) Wang, L.; Nitopi, S. A.; Bertheussen, E.; Orazov, M.; Morales-Guio, C. G.; Liu, X.; Higgins, D. C.; Chan, K.; Nørskov, J. K.; Hahn, C.; et al. ACS Catal. 2018, 8, 7445. doi: 10.1021/acscatal.8b01200

(6) Schouten, K. J. P.; Kwon, Y.; van der Ham, C. J. M.; Qin, Z.; Koper, M. T. M. Chem. Sci. 2011, 2, 1902. doi: 10.1039/c1sc00277e

(7) Montoya, J. H.; Shi, C.; Chan, K.; Norskov, J. K. J. Phys. Chem. Lett. 2015, 6, 2032. doi: 10.1021/acs.jpclett.5b00722

(8) Bertheussen, E.; Verdaguer-Casadevall, A.; Ravasio, D.; Montoya, J. H.; Trimarco, D. B.; Roy, C.; Meier, S.; Wendland, J.; Norskov, J. K.; Stephens, I. E.; et al. Angew. Chem. Int. Ed. 2016, 55, 1450. doi: 10.1002/anie.201508851

(9) Chang, X.; Malkani, A.; Yang, X.; Xu, B. J. Am. Chem. Soc. 2020, 142, 2975. doi: 10.1021/jacs.9b11817 